\title{
DIFERENCIAS LÉXICAS ENTRE EL HOMBRE Y LA MUJER EN TRES CENTROS DE INTERÉS': SALUDOS, TEMAS DE CONVERSACIÓN Y DESPEDIDAS
}

\author{
Gabriela Ríos González.
}

\begin{abstract}
RESUMEN
El siguiente artículo pretende dar a conocer parte de una investigación sobre disponibilidad léxica realizada a jóvenes costarricenses de último año de colegio, que procuró abarcar los diferentes estratos sociales y geográficos del país. En esta ocasión se analizará el lenguaje utilizado por los hombres y las mujeres, se hará una comparación entre los primeros 50 términos en tres centros de interés: Saludos, Temas de conversación y Despedidas.

Palabras clave: Disponibilidad léxica, campos semánticos, lenguaje de género, lenguaje de los jóvenes.
\end{abstract}

\begin{abstract}
The following article looks for explaining a section o for investigation related to Lexicon Availability. This research studied to Costa Rican seniors High School students of the different places of the country and different social status. The investigation analyses the language used by boys and girls making a comparison with the first 50 terms in 3 different interesting centers: greetings, conversation topics and farewells.
\end{abstract}

Key words: Lexicon availability, semantic field, gender's language, young's language.

\begin{abstract}
Adentrarse en el campo de la investigación del género es como entrar en un torbellino. Qué significa ser hembra o macho o cómo es eso de hablar con el otro (o con el mismo) género, son cuestiones cuyas respuestas afectan en carne viva a la gente, y cuando se toca un nervio, la gente chilla. Sin embargo, tengo la esperanza de que a través del estruendo pueda oírse la investigación intelectual erudita y pueda establecerse el diálogo entre los investigadores, incluso entre los que han ingresado en la habitación del intercambio de conocimientos por puertas correspondientes a disciplinas distintas ${ }^{2}$.
\end{abstract}

Gabriela Ríos González. Magíster en Lingüística de la Universidad de Costa Rica. Correo electrónico: gabyrios29@gmail.com,gabyrios29@hotmailcom

Recepción: 24- 3- 2008

Aceptación: 9- 5- 2008 
Este artículo es parte de una investigación más amplia realizada a los jóvenes costarricenses de último año de colegio (aproximadamente en edades comprendidas entre 16 y 18 años), la encuesta fue realizada en todas las provincias del país, aplicada tanto a hombres como a mujeres, en las zonas rural y urbana y en los colegios público y privado. En esta ocasión se analizará únicamente los centros de interés Saludos, Temas de Conversación y Despedidas de un total de 15 centros de interés, pero se espera dar a conocer en próximos artículos algunos resultados de los restantes campos semánticos.

El objetivo principal de este artículo es, apoyados en la metodología de la disponibilidad léxica, dar a conocer las diferencias de vocabulario que existen entre los géneros, específicamente entre los jóvenes de Costa Rica.

Para llevar a cabo la investigación se procedió a realizar un trabajo de campo a la población estudiantil de último año de secundaria de colegios académicos (decimoprimero), en total se encuestó a seiscientos nueve (609) estudiantes, pero se seleccionaron quinientas doce (512) encuestas para ser procesadas, lo que constituyó un 2 por ciento del universo 3 .

La selección fue determinada tomando en cuenta cuatro aspectos:

a. Legibilidad de la encuesta: se eliminaron las encuestas en las cuales no fue posible comprender lo que estaba escrito.

b. Número de encuestas por colegio: algunos centros educativos tenían una extensa población por aula, de tal manera que se eliminaron al azar el número excedido.

c. Encuesta incompleta: se eliminó aquellas encuestas que no fueron completadas en su totalidad, ya sea en los datos del encuestado o propiamente en los datos solicitados.

d. Extranjeros: se excluyeron todas las encuestas de los estudiantes cuyos padres son de otra nacionalidad, para evitar intervenciones léxicas provenientes de otras regiones.

Los centros educativos fueron elegidos tomando en cuenta el tipo de institución pública y privada, la ubicación geográfica urbana y rural y la cantidad de población por provincia; de tal manera que en el siguiente cuadro queda expresada la distribución de la muestra.

Cuadro 1. Desglose de colegios y estudiantes encuestados por público - privado y urbano - rural

\begin{tabular}{lcccccc}
\hline \multicolumn{1}{c}{ Provincia } & \multicolumn{2}{c}{ Público } & \multicolumn{2}{c}{ Privado } & $\begin{array}{c}\text { Total de } \\
\text { Colegios }\end{array}$ & $\begin{array}{c}\text { Número de } \\
\text { estudiantes }\end{array}$ \\
\hline & Urbano & Rural & Urbano & Rural & & \\
\hline San José & 2 & 2 & 2 & & 6 & 128 \\
Alajuela & 2 & 1 & 1 & & 4 & 97 \\
Heredia & 1 & 1 & 1 & & 3 & 68 \\
Cartago & 1 & 2 & 2 & & 5 & 111 \\
Guanacaste & & 1 & & 1 & 2 & 37 \\
Puntarenas & & 1 & & 1 & 2 & 31 \\
Limón & & 1 & & 1 & 2 & 40 \\
\hline TOTAL & 6 & 9 & 6 & 3 & 24 & $\mathbf{5 1 2}$ \\
\hline
\end{tabular}


En el cuadro 1 se muestra el número de colegios encuestados según la provincia, el tipo de centro educativo público o privado y a su vez si estos pertenecen a zona rural o urbana; mientras que las dos últimas columnas expresan la totalidad de colegios por provincia y el total de estudiantes que componen la muestra.

La muestra está conformada por doscientos siete (207) hombres lo que equivale a un 40 por ciento y por trescientos cinco (305) mujeres para un 60 por ciento. Las mujeres superan a los hombres por noventa y ocho (98) informantes, lo que equivale a un 20 por ciento más de mujeres. Este dato se puede justificar, ya que, según el Ministerio de Educación Pública ${ }^{4}$ hay más mujeres que hombres matriculados en el sistema educativo.

La encuesta estuvo constituida por una hoja para que los informantes pusieran sus datos personales, respuestas que pasaron a conformar las variables sociales. Para recopilar la información que sirve de base a esta investigación, se trabajó con 15 centros de interés que se les fueron dando a los informantes una vez concluido el anterior; para cada centro de interés se les dio un tiempo de reacción de dos minutos y para esto nos basamos en el principio de disponibilidad léxica ${ }^{5}$ que afirma que las palabras surgen de forma natural e inmediata alrededor de un tema determinado. El léxico surge espontáneamente cuando existe algo que lo motiva a actualizarse y a fluir por medio de asociaciones. De tal manera que si se le solicita a alguien hablar sobre los Temas de Conversación surgirán de manera espontánea aquellos que están más disponibles en su lexicón mental, en otras palabras, aquellos que utilizan normalmente.

Trataremos de demostrar en este artículo que existen palabras que están más disponibles en la mente de la mujer y otras en la del hombre en tres centros de interés: Saludos, Despedidas y Temas de Conversación.

Es bien sabido que hombres y mujeres hablan de diferente manera, ya sea por el tipo de educación o por cuestiones sociales. Existen algunos lingüistas que se han interesado en el tema y han demostrado que las diferencias entre el lenguaje femenino y el masculino ocurren no solamente en el nivel del léxico, sino también en la fonética y en las diferentes estrategias que utilizan cada uno de ellos en la conversación ${ }^{6}$. En este artículo, sin embargo, competen únicamente las diferencias en el nivel del léxico.

Existen algunas investigaciones sobre disponibilidad léxica en las que las mujeres poseen mayor disponibilidad que los hombres ${ }^{7}$. Sin embargo, no solo el número de palabras producidas es relevante entre el hombre y la mujer, sino también el tipo de léxico empleado ${ }^{8}$, y para tal fin se presenta a continuación tres $\operatorname{listas}^{9}$ (con las primeras cincuenta palabras), una por cada centro de interés comparando hombres y mujeres. Haremos un pequeño análisis de cada una de ellas para luego referirnos de manera general a los datos más relevantes. Iniciaremos por las diferencias léxicas entre géneros en los Saludos.

Cuadro 2. Comparación de léxico, según sexo y centro de interés Saludos ${ }^{10}$

\begin{tabular}{|c|c|c|c|c|}
\hline \multirow[b]{2}{*}{$\mathbf{N}^{\mathbf{o}}$} & \multicolumn{2}{|c|}{ HOMBRES } & \multicolumn{2}{|c|}{ MUJERES } \\
\hline & Palabra & Disponibilidad & Palabra & Disponibilidad \\
\hline 1 & hola & 0.86129 & hola & 0.88493 \\
\hline 2 & ¿cómo estás/tá/tán? & 0.54041 & ¿cómo estás/tá/tán? & 0.63895 \\
\hline 3 & ¿pura vida? & 0.52081 & ¿pura vida? & 0.50560 \\
\hline 4 & ¿qué, mae? & 0.29485 & buenos días & 0.28245 \\
\hline
\end{tabular}


Cuadro 2. Comparación de léxico, según sexo y centro de interés Saludos ${ }^{10}$

\begin{tabular}{|c|c|c|c|c|}
\hline \multirow[b]{2}{*}{$\mathbf{N}^{\circ}$} & \multicolumn{2}{|c|}{ HOMBRES } & \multicolumn{2}{|c|}{ MUJERES } \\
\hline & Palabra & Disponibilidad & Palabra & Disponibilidad \\
\hline 5 & ¿todo bien? & 0.27027 & ¿tuanis? & 0.24821 \\
\hline 6 & buenos días & 0.25422 & ¿todo bien? & 0.24734 \\
\hline 7 & ¿tuanis? & 0.23707 & (i)diay & 0.21511 \\
\hline 8 & ¿qué tal? & 0.17200 & ¿qué tal? & 0.19328 \\
\hline 9 & (i)diay & 0.15213 & buenas noches & 0.18607 \\
\hline 10 & buenas tardes & 0.15121 & buenas tardes & 0.18443 \\
\hline 11 & buenas noches & 0.15033 & hello & 0.16735 \\
\hline 12 & adiós & 0.13786 & adiós & 0.15676 \\
\hline 13 & buenas & 0.13070 & buenas & 0.12770 \\
\hline 14 & ¿cómo (me) le/s va? & 0.10038 & ¿qué, mae? & 0.12296 \\
\hline 15 & ¿bien (bien)? & 0.09012 & ¿cómo (me) le/s va? & 0.10301 \\
\hline 16 & ¿qué, mop? & 0.08742 & ¿cómo (te) va/s? & 0.09772 \\
\hline 17 & hello & 0.07072 & $h i$ & 0.07612 \\
\hline 18 & besos/so & 0.06481 & ¿cómo te/le/s ha ido? & 0.07484 \\
\hline 19 & hasta luego & 0.06085 & ¿bien (bien)? & 0.07317 \\
\hline 20 & abrazos/zo & 0.06077 & besos/so & 0.06891 \\
\hline 21 & ¿qué, huevón?11 & 0.05694 & hey & 0.06377 \\
\hline 22 & ¿cómo (te) va/s? & 0.05372 & abrazos/zo & 0.06211 \\
\hline 23 & hey & 0.04859 & chao/ciao & 0.05822 \\
\hline 24 & chao/ciao & 0.04797 & hasta luego & 0.05776 \\
\hline 25 & ¿qué, loco/ca? & 0.04694 & amigos/go/ga & 0.05297 \\
\hline 26 & amistad/es & 0.04456 & ¿qué, mop? & 0.04618 \\
\hline 27 & ¿cómo te/le/s ha ido? & 0.04309 & tanto tiempo & 0.04315 \\
\hline 28 & $h i$ & 0.04222 & amistad/es & 0.04246 \\
\hline 29 & mae & 0.03990 & ¿(i)diay, qué? & 0.03911 \\
\hline 30 & amigos/go/ga & 0.03983 & ¿qué cuenta/s //contás? & 0.03846 \\
\hline 31 & respeto & 0.03888 & ¿qué has/ha/n hecho? & 0.03604 \\
\hline 32 & ¿qué me dice/s? & 0.03886 & bienvenido/s/da & 0.03230 \\
\hline 33 & (i)diay, mae & 0.03627 & ¿qué me cuenta/s//contás? & 0.03209 \\
\hline 34 & ¿qué? & 0.03601 & bye (bye) & 0.03127 \\
\hline 35 & ¿qué me cuenta/s//contás? & 0.03185 & mae & 0.03124 \\
\hline 36 & mano/s & 0.03096 & $\begin{array}{l}\text { ¿cómo has/ha } \\
\text { estado? }\end{array}$ & 0.02956 \\
\hline 37 & amabilidad & 0.03059 & $\begin{array}{l}\text { ¿cómo amaneció/iste } \\
\text { /ieron /ece? }\end{array}$ & 0.02740 \\
\hline 38 & ¿(i)diay, qué? & 0.02881 & mano/s & 0.02729 \\
\hline 39 & $\begin{array}{l}\text { ¿cómo amaneció/ } \\
\text { iste/ieron/ece? }\end{array}$ & 0.02707 & ¿qué me dice/s? & 0.02513 \\
\hline 40 & ¿(y) entonces (qué)? & 0.02563 & ¿qué hay de nuevo? & 0.02456 \\
\hline
\end{tabular}


Cuadro 2. Comparación de léxico, según sexo y centro de interés Saludos ${ }^{10}$

\begin{tabular}{|c|c|c|c|c|}
\hline & \multicolumn{2}{|c|}{ HOMBRES } & \multicolumn{2}{|c|}{ MUJERES } \\
\hline & Palabra & Disponibilidad & Palabra & Disponibilidad \\
\hline 41 & buenos & 0.02551 & ¿qué? & 0.02374 \\
\hline 42 & ¿qué onda/s? & 0.02225 & cariño/s & 0.02253 \\
\hline 43 & ¿qué cuenta/s//contás? & 0.02220 & respeto & 0.02243 \\
\hline 44 & cortesía & 0.02209 & ¿qué onda/s? & 0.02031 \\
\hline 45 & ¿qué hay? & 0.02162 & buen día & 0.01974 \\
\hline 46 & ¿qué hay de nuevo? & 0.02095 & amor & 0.01911 \\
\hline 47 & ¿cómo has/ha estado? & 0.01964 & ¿cómo se/te encuentra/s? & 0.01843 \\
\hline 48 & $o k(a y)$ & 0.01822 & ¿qué hubo? & 0.01779 \\
\hline 49 & bye (bye) & 0.01812 & cortesía & 0.01713 \\
\hline 50 & ¿qué, compa? & 0.01779 & ¿qué, todo bien? & 0.01707 \\
\hline
\end{tabular}

Al observar el cuadro 2, advertimos que los tres primeros saludos son iguales tanto para los hombres como para las mujeres, los dos primeros son estándares mientras que el tercero es dialectal; hecho que podría llevarnos a suponer que son los más disponibles para la población costarricense en general.

A partir de la posición número cuatro surgen algunas diferencias significativas en cuanto a léxico y a disponibilidad, por un lado las mujeres mencionaron un saludo estándar, mientras que los varones aludieron al término jergal 'mae'12, representado en las mujeres hasta la posición 14 .

En lo que se refiere a los términos que aparecen en una columna y que permanecen ausentes en la otra entre las 50 primeras palabras, notamos que para los hombres inicia en la posición 21 y para las mujeres en la 27. Ahora bien, si nos detenemos a revisar los términos que solo aparecen en los hombres encontramos: ¿qué, huevón?; ¿qué loco/ca?; (i)day, mae; ¿qué hay?; ¿qué, compa? expresiones argóticas que las mujeres no tienen disponible entre sus primeros saludos. Por el contrario, al observar las expresiones usadas por las mujeres, que no tienen disponible los hombres en los primeros saludos, distinguiremos que las palabras o expresiones giran en torno a dos características bien diferenciadas respecto al léxico masculino. Por una parte, abundan los términos del lenguaje estándar sin marcas de tipo diastrático, por ejemplo tanto tiempo, ¿qué has/ha/n hecho?, bienvenido/s/da, buen día, ¿cómo selte encuentra/s?, ¿qué, todo bien? y ¿qué hubo? Por otra parte, muchos de los vocablos o expresiones se insertan en la órbita del afecto. Es el caso de cariño/s y amor.

Otro aspecto que se puede analizar de estas listas es con respecto a los saludos en inglés, aunque los hombres utilizaron un término más que las mujeres (hello, hey, hi, okay y bye), la disponibilidad de las palabras en inglés utilizadas por las mujeres fue mucho mayor que la de los hombres (hello, hey, hi, bye).

En el cuadro 3 distinguiremos los temas de conversación que los estudiantes tienen más disponibles. 
Cuadro 3. Comparación de léxico, según sexo y centro de interés Temas de conversación

\begin{tabular}{|c|c|c|c|c|}
\hline \multirow[b]{2}{*}{$\mathbf{N}^{\circ}$} & \multicolumn{2}{|c|}{ HOMBRES } & \multicolumn{2}{|c|}{ MUJERES } \\
\hline & Palabra & Disponibilidad & Palabra & Disponibilidad \\
\hline 1 & futbol//fútbol & 0.37087 & familia/s & 0.35432 \\
\hline 2 & sexo & 0.35201 & amigos/gas/go & 0.29631 \\
\hline 3 & mujeres/jer & 0.28051 & cole $(\mathrm{gio} / \mathrm{s})$ & 0.26334 \\
\hline 4 & deportes/te & 0.23980 & estudio/s & 0.26079 \\
\hline 5 & cole(gio/s) & 0.23741 & novios/vias/vio/via & 0.23997 \\
\hline 6 & familia/s & 0.22983 & fiestas/ta & 0.22916 \\
\hline 7 & política/co & 0.22643 & chismes/me & 0.22324 \\
\hline 8 & estudio/s & 0.22367 & sexo & 0.20853 \\
\hline 9 & amigos/gas/go & 0.21197 & ropa & 0.20848 \\
\hline 10 & música & 0.20510 & música & 0.20770 \\
\hline 11 & fiestas/ta & 0.20362 & salud & 0.18472 \\
\hline 12 & comida/s & 0.18812 & política/co & 0.16619 \\
\hline 13 & carros/rro & 0.15734 & comida/s & 0.16210 \\
\hline 14 & amor/es & 0.13394 & amor/es & 0.15935 \\
\hline 15 & películas/la & 0.12967 & problemas/ma & 0.15298 \\
\hline 16 & salud & 0.12604 & trabajo/s & 0.14182 \\
\hline 17 & chismes/me & 0.11818 & moda/s & 0.14136 \\
\hline 18 & trabajo/s & 0.11681 & novelas/la & 0.13388 \\
\hline 19 & exámenes/men & 0.10605 & amistad/es & 0.12874 \\
\hline 20 & ropa & 0.09969 & exámenes/men & 0.12547 \\
\hline 21 & novios/vias/vio/via & 0.09739 & hombres/bre & 0.12355 \\
\hline 22 & religión/es & 0.08685 & películas/la & 0.12146 \\
\hline 23 & problemas/ma & 0.08502 & deportes/te & 0.12141 \\
\hline 24 & tele(visión) & 0.08276 & futbol//fútbol & 0.12138 \\
\hline 25 & economía & 0.07394 & sexualidad & 0.09698 \\
\hline 26 & sexualidad & 0.07308 & paseos/seo & 0.09136 \\
\hline 27 & drogas & 0.07150 & vida/s & 0.08957 \\
\hline 28 & noticias & 0.06958 & carros/rro & 0.08059 \\
\hline 29 & futuro & 0.06857 & tele(visión) & 0.07224 \\
\hline 30 & novelas/la & 0.06612 & noticias & 0.06834 \\
\hline 31 & vida/s & 0.05990 & universidad/es & 0.06625 \\
\hline 32 & viajes & 0.05929 & religión/es & 0.06371 \\
\hline 33 & dinero & 0.05624 & profe(sores)s & 0.05967 \\
\hline 34 & amistad/es & 0.05586 & zapatos & 0.05780 \\
\hline 35 & videojuegos/go & 0.05548 & economía & 0.05450 \\
\hline 36 & moda/s & 0.05407 & $\begin{array}{l}\text { programa/s (de) } \\
\text { tele(visión) }\end{array}$ & 0.05447 \\
\hline
\end{tabular}


...continuación

Cuadro 3. Comparación de léxico, según sexo

y centro de interés Temas de conversación

\begin{tabular}{|c|c|c|c|c|}
\hline & \multicolumn{2}{|c|}{ HOMBRES } & \multicolumn{2}{|c|}{ MUJERES } \\
\hline $\mathbf{N}^{\circ}$ & Palabra & Disponibilidad & Palabra & Disponibilidad \\
\hline 37 & tecnología/s & 0.05298 & $\mathrm{casa} / \mathrm{s}$ & 0.05054 \\
\hline 38 & paseos/seo & 0.05249 & enfermedades/dad & 0.05045 \\
\hline 39 & TLC & 0.05020 & animales/mal & 0.04993 \\
\hline 40 & juegos & 0.04704 & drogas & 0.04843 \\
\hline 41 & $\begin{array}{l}\text { programa/s (de) } \\
\text { tele(visión) }\end{array}$ & 0.04555 & futuro & 0.04685 \\
\hline 42 & universidad/es & 0.04366 & educación & 0.04659 \\
\hline 43 & educación & 0.04308 & noviazgo/s & 0.04618 \\
\hline 44 & alcohol & 0.04020 & libros & 0.04445 \\
\hline 45 & pasatiempos/po & 0.03980 & bailes/le & 0.04260 \\
\hline 46 & profe(sores)s & 0.03938 & TLC & 0.04027 \\
\hline 47 & autos & 0.03853 & viajes & 0.03917 \\
\hline 48 & animales/mal & 0.03802 & clima & 0.03916 \\
\hline 49 & sociedad & 0.03593 & mujeres/jer & 0.03861 \\
\hline 50 & casa/s & 0.03535 & cine & 0.03566 \\
\hline
\end{tabular}

El centro de interés Temas de conversación presenta menos palabras diferentes entre géneros, y es a partir de la posición número veintiuno para las mujeres y treinta y tres para los hombres en donde surgen las diferencias, lo que podría llevarnos a interpretar que este centro es más propenso a tener coincidencias en los temas de los cuales hablan los jóvenes costarricenses. Sin embargo, los temas de conversación, en los cuales difieren, muestran estereotipos conversacionales asociados a cada sexo. Creemos, al igual que Lozano que:

\footnotetext{
A través de los siglos, la cultura, en sus diversas formas, se ha encargado de crear una imagen del habla de la mujer que en muchos aspectos pervive en nuestros días... Esta representación social, que se nos va transmitiendo desde la infancia, es la que el individuo se ve obligado a satisfacer comportándose de acuerdo a su sexo tal y como la sociedad espera que lo haga ${ }^{13}$.
}

En este centro de interés las palabras que están en un grupo y en el otro no, son una muestra de los estereotipos conversacionales, temas de conversación que son promovidos por la sociedad como característicos del hombre o la mujer. Por ejemplo los hombres presentaron, en las primeras cincuenta palabras, dinero, videojuegos/go, alcohol, tecnología/s, autos, juegos, pasatiempos/po, y sociedad. Las mujeres por el contrario, hablan de hombres/bre, zapatos, bailes/le, noviazgo/s, enfermedades/dad, libros, clima, cine.

Si comparamos las posiciones de las palabras y su disponibilidad podríamos afirmar que en Costa Rica los hombres tienen temas de conversación que prefieren, mientras que las mujeres tienen otros o con una disponibilidad diferente, merecen especial atención las palabras: chismes, política, ropa, novelas y novios. Pero a pesar de estas diferencias, llama la atención otras que ocupan la misma posición, aunque con diferente disponibilidad: música y amor. 
Opinamos que muchos temas de conversación se han estereotipado y como afirma Lozana (2005) y García Mouton (2003), se han transmitido de generación en generación y que permanecen aún en nuestro tiempo; a pesar de que la mujer trabaja actualmente fuera del hogar y es tan independiente económicamente como el hombre, la familia y la sociedad han sido trasmisoras de lo que puede hablar el hombre y lo que se le permite a la mujer. Esto lo podemos comprobar observando los cuatro primeros campos de los hombres y las mujeres; por un lado, los hombres conversan más sobre: futbol/fútbol, sexo, mujeres/jer, deportes/te; y por el otro las mujeres tienen en su lexicón mental más disponible: familia/s, amigos/gas/go, cole $(\text { gio/s })^{14}$, estudio/s como temas de conversación.

Para finalizar, comparamos en el cuadro 4 las despedidas de los jóvenes costarricenses.

\section{Cuadro 4. Comparación de léxico, según sexo y centro de interés Despedidas}

\begin{tabular}{|c|c|c|c|c|}
\hline & \multicolumn{2}{|c|}{ HOMBRES } & \multicolumn{2}{|c|}{ MUJERES } \\
\hline $\mathrm{N}^{\circ}$ & Palabra & Disponibilidad & Palabra & Disponibilidad \\
\hline 1 & adiós & 0.69130 & adiós & 0.65601 \\
\hline 2 & hasta lueg(uit)o & 0.54048 & hasta lueg(uit)o & 0.49311 \\
\hline 3 & chao (chao)/ciao & 0.41068 & chao (chao)/ciao & 0.48492 \\
\hline 4 & $o k(a y)$ & 0.28596 & bye (bye) & 0.27163 \\
\hline 5 & nos vemos & 0.21629 & tristeza/s & 0.21784 \\
\hline 6 & hasta mañana & 0.19162 & que te/le vaya bien & 0.20773 \\
\hline 7 & bye (bye) & 0.18093 & nos vemos & 0.19512 \\
\hline 8 & hasta pronto & 0.17019 & $o k(a y)$ & 0.19306 \\
\hline 9 & que te/le vaya bien & 0.15999 & hasta pronto & 0.18847 \\
\hline 10 & bueno & 0.15919 & hasta mañana & 0.15685 \\
\hline 11 & tristeza/s & 0.15162 & besos/so & 0.11962 \\
\hline 12 & hasta nunca & 0.14442 & tristes/te & 0.11093 \\
\hline 13 & (de) soltero/ra/ros/ras & 0.08898 & hasta nunca & 0.11068 \\
\hline 14 & tristes/te & 0.08052 & abrazos/zo & 0.10439 \\
\hline 15 & fiesta/s & 0.07927 & dolor & 0.09761 \\
\hline 16 & buenas noches(itas) & 0.07260 & lágrimas/ma & 0.09075 \\
\hline 17 & besos/so & 0.07220 & $\begin{array}{l}\text { (que) Dios lo/te/le/la } \\
\text { acompañe }\end{array}$ & 0.08967 \\
\hline 18 & abrazos/zo & 0.06985 & $\begin{array}{l}\text { cuidate//cuídese/ate } \\
\text { (mucho) }\end{array}$ & 0.08945 \\
\hline 19 & lágrimas/ma & 0.06655 & bueno & 0.08526 \\
\hline 20 & pura vida & 0.05918 & 1lanto/s & 0.07533 \\
\hline 21 & alegría/s & 0.05485 & (de) soltero/ra/ros/ras & 0.07305 \\
\hline 22 & $\begin{array}{l}\text { cuidate//cuídese/ate } \\
\text { (mucho) }\end{array}$ & 0.05164 & buenas noches(itas) & 0.07218 \\
\hline 23 & lo/te/la/los vi & 0.05107 & te quiero (mucho) & 0.07021 \\
\hline
\end{tabular}


Cuadro 4. Comparación de léxico, según sexo y centro de interés Despedidas

\begin{tabular}{|c|c|c|c|c|}
\hline & \multicolumn{2}{|c|}{ HOMBRES } & \multicolumn{2}{|c|}{ MUJERES } \\
\hline $\mathbf{N}^{0}$ & Palabra & Disponibilidad & Palabra & Disponibilidad \\
\hline 24 & lo/te/los/la veo & 0.04982 & llorar & 0.06283 \\
\hline 25 & bueno, mae, $(o k)$ & 0.04803 & alegría/s & 0.05788 \\
\hline 26 & dolor & 0.04646 & fiesta/s & 0.05670 \\
\hline 27 & $\begin{array}{l}\text { (que) Dios lo/te/le/la } \\
\text { acompañe }\end{array}$ & 0.04531 & amor/es & 0.05478 \\
\hline 28 & $\begin{array}{l}\text { (ahí) los/nos/las/les } \\
\text { vidrios }\end{array}$ & 0.04499 & amigos/go/gas & 0.04662 \\
\hline 29 & good bye & 0.04454 & felicidad/es & 0.04465 \\
\hline 30 & recuerdos/do & 0.04311 & chaíto & 0.04056 \\
\hline 31 & amor/es & 0.04081 & viaje/s & 0.03866 \\
\hline 32 & guaro & 0.04079 & se cuida & 0.03754 \\
\hline 33 & sentimientos/to & 0.04034 & good bye & 0.03650 \\
\hline 34 & dolorosas/so/sa/sos & 0.03909 & nostalgia/s & 0.03604 \\
\hline 35 & 1lorar & 0.03877 & soledad & 0.03599 \\
\hline 36 & te quiero (mucho) & 0.03866 & sufrimiento & 0.03538 \\
\hline 37 & 1lanto/s & 0.03736 & $\begin{array}{l}\text { (ahí) los/nos/las/les } \\
\text { vidrios }\end{array}$ & 0.03500 \\
\hline 38 & hasta (la) vista (baby) & 0.03219 & muerte/s & 0.03452 \\
\hline 39 & sexo & 0.03211 & melancolía & 0.03386 \\
\hline 40 & felicidad/es & 0.03098 & alegres/gre & 0.03187 \\
\hline 41 & viaje/s & 0.03077 & recuerdos/do & 0.03163 \\
\hline 42 & alegres/gre & 0.02980 & te amo, (chao) & 0.03107 \\
\hline 43 & soledad & 0.02879 & regalos/lo & 0.03063 \\
\hline 44 & nostalgia/s & 0.02869 & nos vemos luego & 0.02862 \\
\hline 45 & feas/feo & 0.02576 & pura vida & 0.02761 \\
\hline 46 & voy jalando & 0.02552 & dolorosas/so/sa/sos & 0.02726 \\
\hline 47 & me voy & 0.02523 & $\operatorname{amistad} / \mathrm{es}$ & 0.02719 \\
\hline 48 & tuanis & 0.02352 & hasta (la) vista (baby) & 0.02695 \\
\hline 49 & muerte/s & 0.02335 & cariño/s & 0.02630 \\
\hline 50 & hasta (para) siempre & 0.02273 & pórtese//portate bien & 0.02626 \\
\hline
\end{tabular}

Para las Despedidas el panorama es paralelo al campo léxico de los Saludos: los hombres utilizan de nuevo frases del argot juvenil, como bueno, mae (ok); voy jalando; tuanis; y otras que tienen relación con las fiestas de despedida: guaro y sexo. Las mujeres, por el contrario, utilizan en mayor medida las despedidas sin marcas como se cuida, nos vemos 
luego, pórtese//portate bien y expresiones con carga afectiva, que se manifiesta a través de la expresión directa de sentimientos como en los casos de te amo (chao), sufrimiento, melancolía, cariños, o elementos lingüísticos como el diminutivo chaíto.

Al igual que los Saludos, las tres primeras despedidas son iguales para ambos sexos, lo que nos hace pensar que podrían ser las despedidas más utilizadas por la población costarricense.

Después de este pequeño análisis de las tres listas, advertimos que con respecto a las diferencias respecto al número de palabras exclusivas de cada grupo, el centro de interés Despedidas tiene más distancia entre género. En el cuadro 5 resumimos los resultados y presentamos su equivalente en porcentaje. Cuadro 5. Número de palabras exclusivas de
cada grupo para las 50 primeras entradas

\begin{tabular}{cccccc}
\hline \multicolumn{2}{c}{ Saludos } & \multicolumn{2}{c}{ Temas de conversación } & \multicolumn{2}{c}{ Despedidas } \\
\hline Palabra dif. & Porcentaje & Palabra dif. & Porcentaje & Palabra dif. & Porcentaje \\
\hline & & & & & \\
9 & $18 \%$ & 8 & $16 \%$ & 11 & $22 \%$ \\
\hline
\end{tabular}

García Mouton (2003) afirma que un individuo aprende una forma de hablar según unos modelos más o menos flexibles: "Los chicos, a partir de determinada edad, copian modelos masculinos, y las chicas, por su parte, imitan también los femeninos" ${ }^{15}$. La sociedad contribuye - por medio de la educación, de las películas, la publicidad y otros-a formar al individuo en su forma de hablar, creando en muchas ocasiones estereotipos en los que se le permite al hombre cierto comportamiento lingüístico que no se aprueba para las mujeres, o viceversa.

\footnotetext{
Tradicionalmente la mujer ha recibido presiones para que hable poco o para que calle, pero muchas más para que hable "bien" o para que no hable de ciertas cosas: todo esto significa que el lenguaje grosero, las blasfemias y el argot le han estado siempre expresamente prohibidos ${ }^{16}$.
}

Los resultados obtenidos en esta investigación con respecto al tipo de léxico empleado por hombres y mujeres concuerdan con las teorías de que la sociedad le indica al individuo los hábitos lingüísticos y el que las mujeres copian la variante de prestigio.

\footnotetext{
La sociedad educa a los individuos de distinta manera según su sexo, lo que condiciona su posterior comportamiento, al tiempo que se espera que cada uno actúe según esos patrones masculinos o femeninos previamente establecidos. La mujer ha sido tradicionalmente educada e instada a actuar 'como señorita', lo cual implica cierto grado de refinamiento en su comportamiento, sus gestos y ademanes, sus modales de cortesía y, por supuesto, sus hábitos lingüísticos ${ }^{17}$.
}

The common observation that female speakers tend to lead in the use of "prestige" variants ${ }^{18}$.

No se sabe con certeza por qué las mujeres copian las variantes de prestigio, si es porque la sociedad se los impone o por su deseo de "hablar bien", pero es un hecho que la situación se produce entre los jóvenes costarricenses, tal y como asegura García Mouton. 
Los sociolingüistas siempre han afirmado que las mujeres se preocupan más de cómo hablan que los hombres, y que tienden a copiar las costumbres lingüísticas de la gente más educada, de un nivel social superior al suyo. Ellas imitan siempre lo que consideran mejor, de manera que es difícil saber qué empezó antes: si la preocupación de las mujeres por cuidar su lenguaje y por hablar "bien" o su obediencia a los modelos que la sociedad les vende como atractivos y correctos. Lo cierto es que hay formas de hablar que la sociedad identifica como femeninas y que la mujer que se amolda a ellas está mucho mejor considerada que la que no lo hace ${ }^{19}$.

Al mismo tiempo, la mujer busca utilizar el prestigio de la lengua "copiando comportamientos lingüísticos de capas económica o culturalmente superiores a la suya"20; busca la norma e incluso "lo que no es prestigioso en boca de mujer puede llegar a serlo en boca de hombre: en determinados sectores sociales el lenguaje masculino ha tenido tradicionalmente la exclusividad de las palabras groseras, de las blasfemias y del argot, ámbito lingüístico al que la mujer no podría acceder sin sufrir una fuerte sanción" 21.

Esta situación nos remite al concepto de prestigio encubierto, concepto mencionado por primera vez por Trudgill (1971) y retomado por Labov (1983), en el que se afirma que "las mujeres están más influenciadas por las formas estándar que los hombres, pero que los hombres van a la cabeza en el uso de las nuevas formas vernáculas en el discurso casual" 22 .

Se cree que las mujeres se preocupan por utilizar la norma de prestigio porque pretenden el aval social y buscan un estatus frente a los hombres, quienes han disfrutado de un papel social mucho más retribuido y que históricamente han tenido mayor seguridad profesional y personal ${ }^{23}$.

Una vez realizado este pequeño análisis de las diferencias entre el lenguaje de los hombres y las mujeres en los centros de interés: Saludos, Temas de conversación y Despedidas, podemos resumir las conclusiones de la siguiente manera.

En primer lugar, y como lo analizamos anteriormente, destaca el hecho de que ambos sexos comparten el léxico más disponible en los campos Saludos y Despedidas. Esta situación no ha de extrañar ya que nos enfrentamos a centros de interés que incorporan fórmulas que se repiten de manera exacta o casi exacta y que, aunque muestran una gran variedad, como suponíamos en nuestra hipótesis inicial, están extendidas en todos los sociolectos de Costa Rica e incluso en otras diatopías del español. Sería el caso de hola, adiós, ¿cómo estás/táltán?, hasta luego, etc. Sin embargo, observamos que en un significativo tercer lugar encontramos pura vida, que en Costa Rica tiene la misma implantación que los dos primeros sin ningún tipo de marca diastrática.

En segundo lugar, en Temas de conversación a pesar de que la gran mayoría de las entradas son las mismas, para ambos sexos, la disponibilidad es diferente. Esto hace suponer que al mencionar Temas de conversación, los hombres y las mujeres hacen emerger palabras diferentes en los primeros puestos de disponibilidad; quizá debido a que la familia y la sociedad influyen en temas estereotipados y avalan lo que puede ser dicho por las mujeres y por los hombres, o tal vez porque como se dijo en apartados anteriores todo lo que puede ser nombrado es susceptible de ser conversado. De ahí proviene que los primeros cinco temas de mayor disponibilidad para los hombres sean futbol//fútbol, sexo, mujeres/jer, deportes/ te, política/co; mientras que los cinco primeros preferidos por las mujeres sean familia/s, amigos/gas/go, estudio/s, cole(gio/s), novios/vias/vio/via. 


\section{Notas}

1. Conocido también como tema o campo semántico es el "conjunto de expresiones que abarcan un determinado campo de significados; un grupo de expresiones que juntamente con sus significados léxicos o sus contenidos pertenecen a un mismo sistema lingüístico y tienen en común por lo menos un rasgo semántico" Lewandowski (1995: 46-47).

2. Tannen (1996:15).

3. En el momento de la encuesta el universo era de veinticuatro mil doscientos ochenta y siete (24.287) estudiantes en último año, tomando en cuenta colegios públicos y privados, rurales y urbanos.

4. Cfr. página web www.mep.go.cr

5. Propuesto inicialmente por Michéa, Gougenheim, Rivenc y Sauvageot quienes proponen el léxico básico para enseñar francés a los africanos en los años 50. El propulsor de esta metodología para Hispanoamérica ha sido López Morales (1973).

6. Cfr. Lozano (2005: 14).

7. Cfr. Ríos González (2006).

8. Hernández Muñoz (2002) y Sancho Sánchez (2005) hacen una comparación entre los primeros 10 términos empleados por los hombres y las mujeres para cada centro de interés.

9. Las listas están ordenadas por disponibilidad, de tal manera que en el caso de ¿cómo estás/táltán? significa que el primer saludo y con más disponibilidad fue ¿cómo estás?, seguido de ¿cómo está? y por último ¿cómo están? También es importante mencionar que, por ejemplo, ¿pura vida? (0.52081) tuvo más disponibilidad que ¿qué, mae? (0.29485).

10. Los datos marcados con letra negrita son los términos que aparecen en un género, pero que están ausentes en el otro en las primeras 50 entradas.

11. El término gráfico recogido fue güevón.

12. Cuenta con mayor disponibilidad que el equivalente de la misma posición para las mujeres.

13. Lozano (2005: 19).

14. El paréntesis significa optativo, la palabra cole(gio/s) debe interpretarse que fue mencionada como cole, colegio y colegios.

15. García Mouton (2003: 23).

16. García Mouton (2003:103).

17. Lozano (2005: 144). 
18. Eckert (2000: 120).

19. García Mouton (2003: 24).

20. García Mouton (1999: 52).

21. García Mouton (1999: 52-53).

22. Labov (1983: 374).

23. Cfr. García Mouton (1999: 53).

\section{Bibliografía}

Aijón Oliva, Miguel Ángel. 2003. La situación comunicativa: propuesta de un modelo formal sociolingüístico. Trabajo de Grado: Universidad de Salamanca (inédito).

Areiza Londoño, Rafael. 2005. "iPrimero se saluda!: Una mirada sociolingüística al saludo en el Eje Cafetalero (Colombia)”. En: Murillo (ed.), 85-95.

Arrieta, Marjorie et al. 1986. "Actitudes lingüísticas hacia dos variedades de habla: Valle Central y Guanacaste”. Revista de Filología y Lingüística. 12 (2): 113- 128.

Avelló Flórez, José y Antonio P. Muñoz-Carrión. (1989). "Cultura juvenil: La comunicación desamparada". En: Rodríguez (coord.), 23- 54.

Ávila, Raúl et al. 2003. Pautas y pistas en el análisis del léxico hispano (americano). Alemania: Vervuert.

Bartol Hernández, José Antonio. 2003. "Léxico disponible y norma lingüística". En: Moreno Fernández et al. (coords.). Vol. I, 127- 144.

2004. Léxico disponible de Soria. Estudio y Diccionarios. Burgos: Fundación Instituto Castellano y Leonés de la Lengua.

Benítez Pérez, Pedro. 2003. "Consideraciones en torno a la enseñanza del vocabulario". En: Moreno Fernández et al. (coords.). Vol. I, 145- 156.

Borrego Niego, Julio y Carmen Fernández Juncal. 2000. "Léxico disponible: aplicaciones a los estudios dialectales". En: Muñoz Núñez et al. (eds.). IV Congreso de Lingüística General. Vol II, 297- 306.

2003. "En qué cambia la universidad la disponibilidad léxica de los preuniversitarios?”. En: Moreno Fernández et al. (coords). Vol. I, 167- 178. 
Borrego Nieto, Julio. 2004. "Algunas preguntas en relación con el concepto de 'Léxico disponible"”. En: Prado Aragonés y Galloso Camacho (eds.), 59- 69.

Camacho Víquez, Hilda. 1997. Léxico del español del Valle Central de Costa Rica en los campos deferenciales alimentación, casa, cuerpo humano, salud, familia, ciclo de vida, vestuario, vida social, y diversiones. Tesis de Licenciatura en Filología Española: Universidad de Costa Rica.

Cárcedo González, Alberto. 1998. “Tradición y novedad en las aportaciones hispánicas a los estudios de disponibilidad léxica”. Lingüística. Asociación de Lingüística y Filología de la América Latina (ALFAL). 10: 5- 68.

2003. "Unidad y variedad diatópico de la disponibilidad léxica del español: comparación de los inventarios de Puerto Rico, Cádiz y Asturias". En: Moreno Fernández et al. (coords.). Vol. I, 199- 225.

Casado Velarde, Manuel. 1989. "Léxico e idiología la lengua juvenil”. En: Rodríguez (coord.), 167- 178.

2002. "Aspectos morfológicos y semánticos del lenguaje juvenil". En: Rodríguez (coord.), 57- 66.

Català, Natàlia. 1989. “Consideraciones acerca de la pobreza expresiva de los jóvenes”. En: Rodríguez (coord.), 203- 216.

Costa, Pere-Oriol, José Manuel Pérez y Fabio Tropea. 1997. Tribus urbanas. El ansia de identidad juvenil: entre el culto a la imagen y la autoafirmación a través de la violencia. Barcelona: Paidós.

Eckert, Penelope. 2000. Linguistic variation as social practice. England: Stanford University.

Elizondo Morales, Mainor. 1996. "Vocabulario del cantón de San Ramón de Alajuela". Revista de Filología y Lingüística. 22 (2): 143- 178.

García Mouton, Pilar. 1999. Cómo hablan las mujeres. Madrid: Arco Libros.

Hernández Muñoz, Natividad y Julio Borrego Nieto. 2004. “Cuestiones metodológicas sobre los estudios de disponibilidad léxica". En: Villayandre Llamazares (ed.), 1519- 1527.

Hernández Muñoz, Natividad. 2005. Hacia una teoría cognitiva integrada de la disponibilidad léxica: El léxico disponible de los estudiantes castellano-manchegos. Tesis de Doctorado: Universidad de Salamanca, España. 
Labov, William. 1983. Modelos sociolingüísticos. Madrid: Cátedra.

Lewandowski, Theodor. 1992. Diccionario de Lingüística. García-Denche y Bernárdez (trads.). Madrid: Cátedra.

López Morales, Humberto. 1986. Enseñanza de la lengua materna. Madrid: Playor.

1993. “Los estudios de disponibilidad léxica: pasado y presente”. En: Sánchez Corrales (ed.), 29- 40 .

1998. La aventura del español en América. Madrid: Editorial Espasa.

1999. Léxico disponible de Puerto Rico. Madrid: Arco Libros.

Lozano Domingo, Irene. 2005. Lenguaje femenino, lenguaje masculino. ¿Condiciona nuestro sexo la forma de hablar? Madrid: Minerva Ediciones.

Moreno, Francisco. 1989. “Elementos no marginales en la lengua coloquial de los jóvenes”. En: Rodríguez (coord.), 241- 270.

Moreno Fernández, F. et al. (coords.). 2003. Lengua, variación y contexto. Estudios dedicados a Humberto López Morales. Madrid: Arco Libros.

Murillo, Jorge (edit.). 2005. II Coloquio Internacional del Programa EDICE. San José, Costa Rica: Editorial de la Universidad de Costa Rica.

Prado Aragonés, Josefina y Ma. Victoria Galloso Camacho (eds.). 2004. Diccionario, Léxico y Cultura. Huelva: Publicaciones Universidad de Huelva.

Quesada Pacheco, Miguel Ángel. 1991, 2001. Nuevo diccionario de costarriqueñismos. $3^{\mathrm{a}}$ ed. Costa Rica: Editorial Tecnológica de Costa Rica.

Rivière, Margarita. 1989. "Moda de los jóvenes: un lenguaje adulterado”. En: Rodríguez (coord.), 71- 77.

Rodríguez González, Félix (coord.). 1989. Comunicación y lenguaje juvenil. Madrid: Editorial Fundamentos.

1989. “Lenguaje y contracultura juvenil: anatomía de una generación”. En: Rodríguez (coord.), 135- 166.

Rodríguez González, Félix (coord.). 2002. El lenguaje de los jóvenes. Barcelona: Editorial Ariel, S.A. 
Samper Padilla, José Antonio. 1998. "Criterios de edición del léxico disponible: sugerencias”. Lingüística. Asociación de Lingüistas y Filólogos de América Latina (ALFAL). 10: 311- 333.

Samper Padilla, José Antonio; Juan José Bellón Fernández y Marta Samper Hernández. 2003. "El proyecto de estudio de la disponibilidad léxica en español". En: Ávila et al.

Sánchez Corrales, Víctor (ed.). 1993. Memorias del IV Congreso costarricense de Filología, Lingüística y Literatura. San José: Oficina de Publicaciones de la Universidad de Costa Rica.

Tannen, Deborah. 1996. Género y discurso. Barcelona: Paidós.

Villayandre Llamazares, Milka (ed.). 2004. Actas del V Congreso de Lingüística General. Madrid: Arco Libros

Zimmermann, Klaus. 2002. "La variedad juvenil y la interacción verbal entre jóvenes”. En: Rodríguez (coord.). Barcelona: Ariel, 137- 163. 